\title{
Classification, Gender and Age Differences, and Seasonal Changes in Relation to Patterns of Distribution Curves for Physique and Motor Performance in Preschool-Aged Japanese Children
}

\author{
Takahiro Ikeda $^{1}$ and Osamu Aoyagi ${ }^{2}$ \\ 1. School of Human and Social Sciences, Fukuoka Prefectural University, Tagawa, Fukuoka 825-8585, Japan \\ 2. Faculty of Sports and Health Science, Fukuoka University, Jonan-ku, Fukuoka 814-0180, Japan
}

\begin{abstract}
It is predicted that the distribution on score of children's motor performance show abnormal curve, because their physical fitness shows decline and gap between good and poor. The aim of present study is to type pattern of distribution curve on physique and motor performance (PMP) in preschoolers, and to investigate gender difference, and change in season and age on distribution of PMP. Seven hundred and seven preschoolers participate in measurement on 21 items of PMP. And mix-longitudinal data which made from 6 terms ( 2 seasons of spring and autumn for 3 years) is completed. The histograms of 10 sections are trained for each gender, term, and test item based on range of data including mean \pm 3 standard deviation. By cluster analysis and multi-correspondence analysis, "physique \& jumping" show closely normal curve, but "manipulation (MP)" is skewed to poor and "running \& prompt (RP)" is skewed to good. By Hotelling's T2-test and Mahalanobis' Distance, gender difference is shown that boys' RP is skewed to good, and their "throwing" and "weight \& flexibility (WF)" are skewed to poor. About season, WF is skewed to poor and RP is skewed to good, in spring. In annual change about pattern of distribution, skewing poor in MP and good in "running straight \& prompt" are changed to normal curve with aging.
\end{abstract}

Key words: Distribution, gap of motor ability, Hotelling's T2-test, Mahalanobis' Distance, multi-correspondence analysis.

\section{Introduction}

Although improvements have been seen in physical measurements among Japanese children in recent years, a steady decrease has been seen in motor performance, which suggests that children's attitudes toward activities that maintain and improve their physical fitness and motor ability have become polarized; that is, they tend to be either extremely active or extremely inactive [1]. Due to this polarization, conventional measurement methods that focus solely on changes in mean values have become insufficient for grasping the actual status and changes in the physical fitness and motor ability of children [2]. Changes in the shape of distribution curves based on

Corresponding author: Takahiro Ikeda, Ph.D., professor, research field: human growth and development. means and standard deviations (SD) for endurance running tests in 17-year-olds have been reported in previous studies [3, 4]. In those studies, their means declined and their distribution curves became smooth. This smoothing suggested that the "gap" in their measurements was becoming wider. Matsuura [5] reported that physical features in humans typically have a normal distribution, and Ohshima [6] found that the disturbance of distribution indicates a degree of abnormality in a group. These findings lead us to suspect that some issues regarding physical fitness and/or motor ability recently seen among children will also appear in the distribution of their physical features in some form or another. In order to grasp the actual situation, an examination of distribution patterns for these measurements is necessary. 
The Ministry of Education, Culture, Sports, Science and Technology in Japan recently debated the importance of physical activities, such as motor play, in which children independently move their bodies in daily life throughout childhood, and subsequently published Exercise Guidelines in Young Childhood [7]. Moreover, Sugihara et al. [8] suggested that the change in physical fitness/motor abilities was identical in preschool-aged children and their older counterparts. The gaps in physical fitness/motor abilities or the amount of physical activities that children participate in at a younger age affect their future health conditions and level of physical fitness [9]. This suggests the importance of dealing with the deterioration of young children's physical fitness/motor abilities from early childhood. Furthermore, some studies have reported differences in the distributions of measurements for motor performance in young children [10-12]. Ikeda and Aoyagi $[10,11]$ investigated distributions of motor performance using skewness and kurtosis statistics from 20 separate performance tests, and based on estimates after defining the polarization by kurtosis, the characteristics of polarized motor performance. Kasuga et al. [12] investigated changes with age in histograms of seven physical fitness and motor abilities by gender based on data comprising more than 5000 young children. However, while Ikeda and Aoyagi [10, 11] grouped children by actual age, Kasuga et al. [12] did so by every half year of age, and thus their groupings were not based on academic year, which was more typical at child care and educational sites observed in this study. The children observed in this study were usually classified by Japanese academic year, which begins in April and ends in March. Therefore, analyses were based on the assumption that the polarization of the decline of physical fitness and/or physical activities would affect to some degree the distribution curve for motor performance. Therefore, in this study, the grouping of children by school year was considered reasonable. In addition, seasonal changes are known to greatly influence the physical activities of young children [13, 14]. As childhood is a period of remarkable growth and development, changes should be observed semi-annually. Furthermore, gender differences in motor performance during childhood have been reported [15]. It is expected that the gender differences found in physical fitness and/or motor abilities cause some impact on the shape of distribution. The aims of this study were to investigate the shape and to classify the patterns of distribution for physique and motor performance (PMP) in childhood and to examine gender differences and seasonal changes in PMP distributions for different ages.

\section{Methods}

\subsection{Samples}

The study participants were 707 preschoolers (352 boys, 355 girls; 207 junior class, 246 middle class, and 254 senior class pupils). All participants completed physique measurements and motor performance tests. Measurements were made twice a year, in May (spring) and November (autumn), and these measurements were sustained for two years. As shown in Table 1, the students measured as junior class pupils during the first year were measured as middle class pupils during the second year, and so on. All students attended four measurements in two years. In short, although some data are missing due to student transfers or dropouts, a mixed longitudinal data set [16] for two or four terms allows the following six-term changes to be observed: junior class/spring (T1); junior class/autumn (T2); middle class/spring (T3); middle class/autumn (T4); senior class/spring (T5); and senior class/autumn (T6). No pupils diagnosed with or suspected of having developmental disorders were included in the analysis.

\subsection{Test Items}

Standing height and body weight were measured to 
Table 1 Outline of the study participants and parameters.

\begin{tabular}{|c|c|c|c|c|c|c|c|}
\hline \multirow[b]{2}{*}{ Year } & \multirow[b]{2}{*}{ Season } & \multicolumn{2}{|c|}{ Junior class } & \multicolumn{2}{|c|}{ Middle class } & \multicolumn{2}{|c|}{ Senior class } \\
\hline & & Boys & Girls & Boys & Girls & Boys & Girls \\
\hline \multirow{2}{*}{ First } & Spring & 48 & 51 & 60 & 66 & 65 & 58 \\
\hline & Autumn & 48 & 51 & 60 & 66 & 65 & 58 \\
\hline \multirow{2}{*}{ Second } & Spring & 52 & 53 & 53 & 64 & 62 & 63 \\
\hline & Autumn & 53 & 53 & 53 & 64 & 66 & 63 \\
\hline \multirow{2}{*}{ Subtotal for 2 years } & Spring & 100 & 104 & 113 & 130 & 127 & 121 \\
\hline & Autumn & 101 & 104 & 113 & 130 & 131 & 121 \\
\hline Subtotal for grade & & Junior & 207 & Middle & 246 & Senior & 254 \\
\hline Subtotal for gender & & & & Boys & 352 & Girls & 355 \\
\hline
\end{tabular}

Table 2 Physique and motor performance (PMP) test items.

\begin{tabular}{|c|c|c|c|}
\hline No. & Item & Unit & Method \\
\hline 1 & Height & $\mathrm{cm}$ & Regular method \\
\hline 2 & Weight & $\mathrm{kg}$ & Regular method \\
\hline 3 & 25-m run & $1 / 10 \mathrm{~s}$ & Two children run $30 \mathrm{~m}$ together (elapsed time to $25 \mathrm{~m}$ was noted) \\
\hline 4 & Potato race & $1 / 10 \mathrm{~s}$ & Duration of time to retrieve three bean bags one by one ( $3 \mathrm{~m}$ away) \\
\hline 5 & Side jumps & times & Number of side-jumps between 30 -cm wide parallel lines \\
\hline 6 & Vertical jump & $\mathrm{cm}$ & Distance reached when jumping from a standing position \\
\hline 7 & Standing broad jump & $\mathrm{cm}$ & Distance of jumping \\
\hline 8 & Jumping back and forth & times & Same as item 5, but jumping back and forth \\
\hline 9 & Ken-ken-pa & $1 / 10 \mathrm{~s}$ & $\begin{array}{l}\text { Duration of time to perform three double hops and jumps within } \\
40 \text {-cm-diameter circles }\end{array}$ \\
\hline 10 & Overhead throw with both hands & $0.5 \mathrm{~m}$ & Distance a ball is thrown (overhead with both hands like a soccer throw-in) \\
\hline 11 & Tennis ball throw & $0.5 \mathrm{~m}$ & Distance a tennis ball is thrown (overhand throw) \\
\hline 12 & T-ball & $\mathrm{m}$ & Distance of hitting a t-ball with a bat \\
\hline 13 & Kick for distance & $0.5 \mathrm{~m}$ & Distance reached by a kicked ball \\
\hline 14 & Rolling hoop & $0.5 \mathrm{~m}$ & Distance reached when a 70-cm-diameter hoop was rolled \\
\hline 15 & Balance beam walk & $1 / 10 \mathrm{~s}$ & Time to walk between two stands located at opposite ends of a balance beam \\
\hline 16 & Jump over and crawl under & $1 / 10 \mathrm{~s}$ & $\begin{array}{l}\text { Duration of time to jump over and crawl under a } 30-\mathrm{cm} \text {-high elastic string } \\
\text { (three times) }\end{array}$ \\
\hline 17 & Hurdle run & $1 / 10 \mathrm{~s}$ & $\begin{array}{l}\text { Duration of time to run and jump a } 12-\mathrm{m} \text { course with six obstacles set at } 2-\mathrm{m} \\
\text { intervals }\end{array}$ \\
\hline 18 & Stand up and dash & $1 / 10 \mathrm{~s}$ & $\begin{array}{l}\text { Duration of time to stand from a prone position, run to, run around, and } \\
\text { return from a target } 3 \mathrm{~m} \text { away }\end{array}$ \\
\hline 19 & Mini-putt golf & $0.5 \mathrm{~m}$ & Distance obtained when putting towards a $10-\mathrm{m}$ target \\
\hline 20 & Sit-and-reach & $\mathrm{cm}$ & Distance reached while sitting on floor, legs extended, reaching towards toes \\
\hline 21 & Total reaction time & $0.001 \mathrm{~s}$ & Jumping reaction time after indication by a light signal \\
\hline
\end{tabular}

classify physique. Traditional tests widely used for children in Japan and new tests developed based on the Fundamental Motor Skill Model by Gallahue and Donnelly [17] were used to assess motor performance. Tests were composed of 19 items that had been verified for reliability, validity, and practicability by Ikeda and Aoyagi [18]. The test items and their measurement procedures are shown in Table 2 . Measurements were conducted by the following types of testers: (1) a researcher at a higher educational institute specializing in the growth and development of young children; (2) university students who attended a lecture on the measurement of physical fitness in young children; and (3) kindergarten teachers whose kindergarten pupils were measured.

\subsection{Statistical Analysis}

Obtained data were aggregated in six terms ( $\mathrm{T} 1$ to 
T6) combined with gender (boys and girls) and grade (junior, middle and senior classes). To remove extremes, only data having values within 3 SD greater or less than the mean were used. The number of class intervals was then uniformly divided into 10 sections (S1 to S10) for all items. Next, using the criterion of $5 \%$ in each class interval, the deviations of obtained and normal distributions were classified into the following three patterns: deviations in the convex $(+)$ and concave (-) directions, and no deviations within $5 \%$ (even) (Fig. 1). No concave direction (-) was found in S1, S2, S9 or S10 class intervals; however, for observing the polarization in distribution of motor performance, only convex (+) and no deviations (even) were examined in these classes. Next, the deviation patterns $(+,-$, even) regarding the gender and grades classifications of preschoolers were analysed based on multiple correspondence analysis [19]. Types of category scores (CSs) of convex (+), concave (-), and no deviations (even) were plotted in a two-dimensional configuration with varimax rotation. After the rotation, the means of object scores (OSs), which were rotated in the same manner, were also superimposed onto a two-dimensional configuration. Then, based on the means of OSs for each test item, hierarchical cluster analysis was conducted to classify motor performance. The distance between clusters was defined by the average linkage method. PMP was clustered based on the shape of dendrogram. Motor performance was then roughly classified into upper and
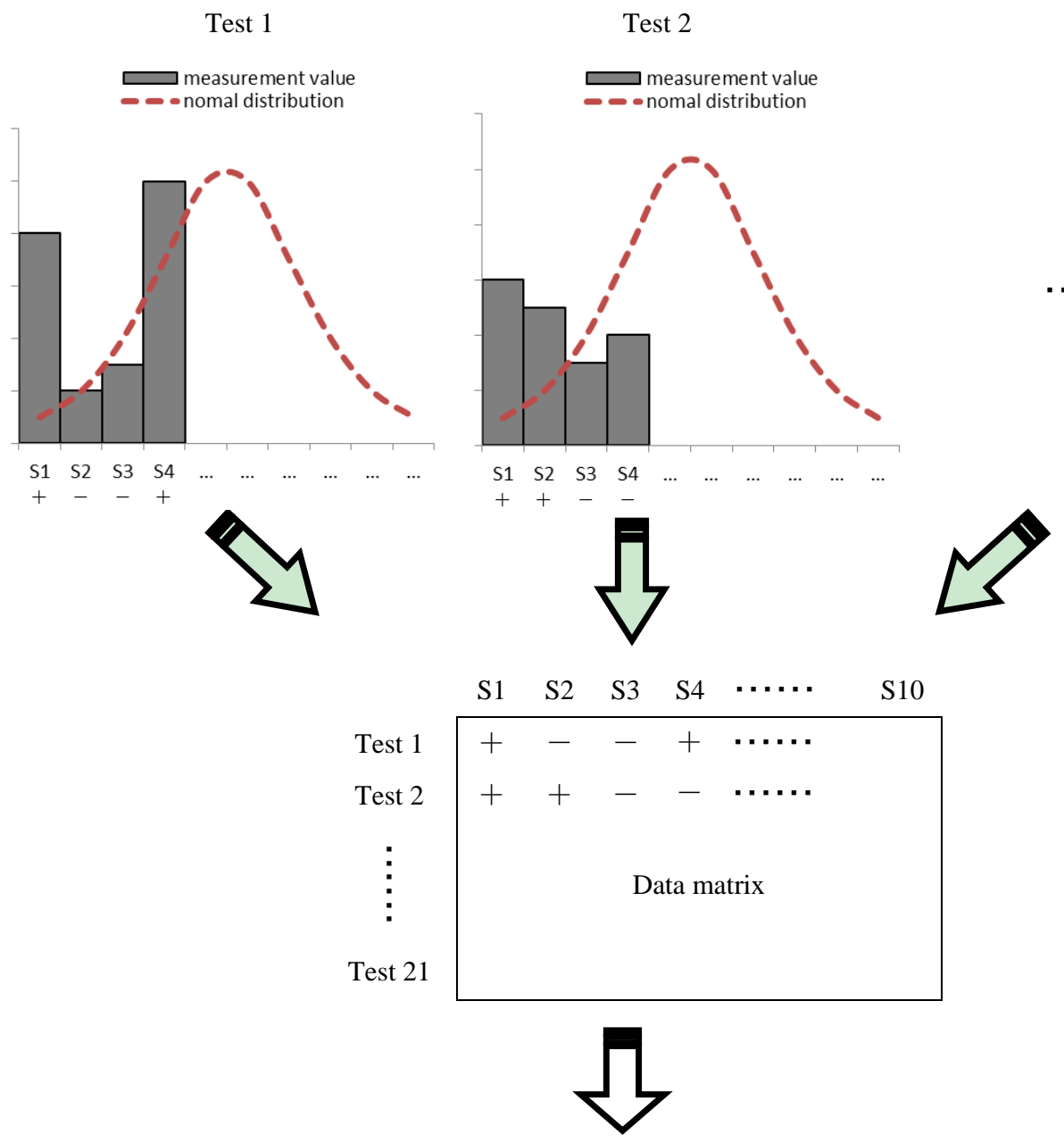

Multi-correspondence analysis \& cluster analysis

Fig. 1 The pattern diagram of analyzing data. 
lower clusters. Next, differences between the means of OSs for clustered items were analysed based on the change and pattern of distributions, and the differences in means among the upper clusters were tested. Gender and seasonal changes were expressed as two variables in the first and second dimensions, and Hotelling's T2 test [19] was used for analysis. Then, to investigate sub-clusters among clusters that showed a significant difference, generalized Mahalanobis distance [19] was used. At the same time, characteristics of the differences and directional changes were observed in a two-dimensional configuration after plotting the means of OSs. For this study, all significance levels for statistical testing were set at 5\%. Generalized Mahalanobis distance is equal to the square of the effect size (Cohen's d), which was advocated by both Cohen [20] and Thomas and French [21, 22]; therefore, the present study regarded the value of "0.04-0.25" as "small", " $0.25-0.64$ " as "medium", and "0.64 or greater" as "large".

\subsection{Ethical Considerations}

Before the study began, consent to participate was obtained from the guardians of all the children after they received written explanations regarding the study purposes. For children who were considered difficult to measure for various reasons, and for those who expressed their unwillingness to take part were not forced to participate.

\section{Results}

\subsection{PMP Statistics}

After computing the means and SD of PMP tests by gender and term, physical growth and development in physique and function tended to increase with increasing age in most of the items. Growth and development were also in items not showing the same trend, but these changes were not significant. The following items did not fall within $3 \mathrm{SD}$ from the mean in four children or more: "Potato race (boys in T3 and girls in T4)"; "Ken-ken-pa (hop, step and land on both feet, boys in T5)"; "Jump over and crawl under (boys in T4)"; and "Balance beam walk (girls in T3)". The items that had the most outliers (five children) were "25-m run (boys in T5)", "Total reaction time (boys in T5)", and "Balance beam walk (girls in T4)". However, the proportion of outliers in all items for the entire sample was less than $4 \%$.

\subsection{Deviation of Distributions and Classification of Motor Performance}

After categorizing the \pm 3 SD range into 10 sections by gender and item, which were set from S1 with poor PMP to S10 with excellent PMP, any observed deviations and directions were compared with a normal distribution on the basis of each section as 5\%. No deviations were found in S1, S2, S9 and S10 for all measurement items in both genders and terms. There, it was decided to focus on sections S3 to S8 in a later analysis. Table 3 shows the deviations among sections $\mathrm{S} 3$ to $\mathrm{S} 8$ in each item by gender as compared with normal distributions. Based on the results, a multiple correspondence analysis was conducted to interpret the overall characteristics of the distribution by setting the presence/absence or directions of deviations as a categorical variable. CSs plotted in a two-dimensional configuration with varimax rotation are shown in Fig. 2. In the first dimension, concave (-) CSs relating to S4, S5 and S8 were plotted in a positive direction (right side), while convex (+) CSs relating to S6 and S7 were plotted in a negative direction (left side). In the second dimension (upper side), convex (+) CSs relating to S4 and S5, and concave (-) CSs relating to S6 and S7 were plotted in a positive direction, and no deviations (even) from S3 to S8 were plotted in a negative direction. Therefore, CSs in the first dimension were "skewed to good", and CSs in the second were "skewed to poor." 
Table 3 Deviations among sections in each item by gender as compared with normal distributions

\begin{tabular}{|c|c|c|c|c|c|c|c|c|c|c|c|c|c|c|c|c|c|c|c|c|c|c|}
\hline \multicolumn{2}{|c|}{ Item no. ${ }^{1}$} & 1 & 2 & 3 & 4 & 5 & 6 & 7 & 8 & 9 & 10 & 11 & 12 & 13 & 14 & 15 & 16 & 17 & 18 & 19 & 20 & 21 \\
\hline \multirow{6}{*}{$\mathrm{T} 1$} & S3 & + & & & & & & & & - & - & & & & - & & & & & & & \\
\hline & S4 & - & & - & & & & & & - & + & + & + & + & + & - & & - & - & - & + & \\
\hline & S5 & & & & & + & & & + & - & + & & & + & + & & & - & & + & & \\
\hline & S6 & & & + & + & & & & & + & + & + & + & - & & + & + & + & + & & & + \\
\hline & S7 & & - & + & + & - & + & & - & + & - & & - & - & - & + & + & + & + & & - & + \\
\hline & S8 & & & - & - & & & & & - & & - & & . & & - & - & - & - & & & \\
\hline \multirow{6}{*}{$\mathrm{T} 2$} & S3 & & & & & & & & & & + & & + & - & - & & & & & & & \\
\hline & S4 & & & & & & & & + & - & - & + & & + & + & - & - & - & - & - & & \\
\hline & S5 & & & - & & + & & & + & & & + & + & - & + & & & - & & + & & - \\
\hline & S6 & + & & & & - & - & & & + & & & - & - & & + & & + & & & & \\
\hline & S7 & & - & + & + & - & & & - & + & & - & & & - & + & + & + & + & - & & + \\
\hline & S8 & & & & - & & & & & - & & & & - & & - & - & - & & + & & \\
\hline \multirow{6}{*}{$\mathrm{T} 3$} & S3 & & & & & & & & & & & + & - & & - & - & & - & & & & \\
\hline & S4 & & & - & - & & & & + & & & - & + & + & + & - & & - & - & & & \\
\hline & S5 & + & + & - & & & & & - & - & & + & & & + & & - & - & & - & + & - \\
\hline & S6 & & & + & + & & & & & + & & & & - & & + & + & + & + & + & & + \\
\hline & S7 & & - & + & + & & & & & + & & - & - & - & - & & + & & & & - & + \\
\hline & S8 & & & - & - & & & & & - & & & & & & - & - & - & - & & & - \\
\hline \multirow{6}{*}{$\mathrm{T} 4$} & S3 & & & - & & & & & & - & & & & & & - & & & & & & \\
\hline & S4 & & & & - & & & & & - & & & & & + & - & - & - & & & & \\
\hline & S5 & & + & & - & & & & & & & + & & & & - & - & & & & & \\
\hline & S6 & & & + & + & & & & - & + & & & & + & & + & + & & + & & - & \\
\hline & S7 & & - & & & & & - & & & & - & + & - & - & & + & & & + & & + \\
\hline & S8 & & & & & & & & & & & & - & - & & - & - & & & & & - \\
\hline \multirow{6}{*}{ T5 } & S3 & & & & - & & & & & - & & & - & + & & & - & - & - & & & \\
\hline & S4 & & & - & - & - & & & & & + & & + & & + & - & - & - & - & & & \\
\hline & S5 & & & + & - & & & + & + & & & + & & & + & & & & + & - & & \\
\hline & S6 & & & + & + & & + & & & + & & & & & - & + & + & + & + & + & & + \\
\hline & S7 & & - & + & - & & - & & & + & & - & - & & - & + & & + & & + & & + \\
\hline & S8 & & & - & - & & & & & - & & & & & & - & - & - & - & & & \\
\hline \multirow{6}{*}{ T6 } & S3 & & & & - & & & & & & & & & & & & & & & & & \\
\hline & S4 & & & - & - & & - & & & - & & & + & & + & - & - & - & & & + & \\
\hline & S5 & & & & & & + & & & & + & & & + & & & - & & & - & & - \\
\hline & S6 & & & + & + & & + & & & + & - & & & - & & + & + & + & & & & + \\
\hline & S7 & & & + & + & & & & & + & - & - & - & - & & & & & & + & - & + \\
\hline & S8 & & & & - & & & & & & + & & & & & - & - & - & & & & \\
\hline
\end{tabular}


(Table 3 Continued)

Girls

\begin{tabular}{|c|c|c|c|c|c|c|c|c|c|c|c|c|c|c|c|c|c|c|c|c|c|c|}
\hline \multicolumn{2}{|c|}{ Item no. } & 1 & 2 & 3 & 4 & 5 & 6 & 7 & 8 & 9 & 10 & 11 & 12 & 13 & 14 & 15 & 16 & 17 & 18 & 19 & 20 & 21 \\
\hline \multirow{6}{*}{$\mathrm{T} 1$} & S3 & & & & & & & \multirow{6}{*}{+} & \multirow{6}{*}{+} & & + & \multirow{6}{*}{-} & \multirow{6}{*}{$\overline{+}$} & & - & & & & & & \multirow{6}{*}{$\begin{array}{l}+ \\
-\end{array}$} & \multirow{6}{*}{+} \\
\hline & S4 & + & & - & - & - & - & & & - & + & & & - & + & - & & - & - & & & \\
\hline & S5 & & & & - & + & + & & & & - & & & + & & & & & - & & & \\
\hline & S6 & & & + & + & & & & & + & + & & & + & & + & & + & + & & & \\
\hline & S7 & & & & + & & & & & + & & & & - & - & + & + & + & + & & & \\
\hline & S8 & & & - & - & & & & & & - & & & & & - & & - & - & & & \\
\hline \multirow{6}{*}{$\mathrm{T} 2$} & S3 & & & \multirow{6}{*}{+} & - & & & & & & + & & - & - & - & & . & - & & & & - \\
\hline & S4 & & & & - & & & & & - & - & & + & + & + & - & & - & - & & & \\
\hline & S5 & & & & & & + & & & & + & - & + & & + & & - & - & & & - & \\
\hline & S6 & & & & + & & & & - & + & - & & & & - & - & + & + & & & & + \\
\hline & S7 & & & & & - & - & & + & + & + & + & - & - & - & + & + & & + & & & \\
\hline & S8 & & & & - & & & & & - & & & - & + & & - & & - & & & & - \\
\hline \multirow{6}{*}{ T3 } & S3 & & & \multirow{6}{*}{+} & & & & \multirow{6}{*}{-} & & & & + & - & & - & - & . & - & & & & 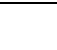 \\
\hline & S4 & & & & - & + & & & + & & & - & + & + & + & - & - & - & - & - & + & - \\
\hline & S5 & & + & & & & - & & & & & + & - & & & - & & & & & & - \\
\hline & S6 & & - & & + & - & + & & - & + & & - & - & & - & + & + & + & + & & & + \\
\hline & S7 & & & & + & & & & + & & & + & & - & - & + & + & + & + & & & + \\
\hline & S8 & & & & - & & & & & & - & & & & & - & & & & & & \\
\hline \multirow{6}{*}{ T4 } & S3 & & & \multirow{4}{*}{-} & & & & & & & + & & & - & - & - & & - & & & & \\
\hline & S4 & & & & - & & & & - & - & + & - & + & + & + & - & & & - & & & - \\
\hline & S5 & & & & & & & & - & - & - & + & & + & + & - & & & & & & \\
\hline & S6 & & - & & + & & & & & + & + & - & - & & - & + & & + & + & & & + \\
\hline & S7 & & & + & + & & & & + & + & & + & & - & & - & + & + & + & & & + \\
\hline & S8 & & & & - & - & & & & & & & & & & - & & & & & & \\
\hline \multirow{6}{*}{ T5 } & S3 & & & \multirow{6}{*}{+} & - & + & & & & - & & & & & & & & & & & & \\
\hline & S4 & & & & - & - & & & - & - & & & & + & + & - & - & & - & - & & \\
\hline & S5 & & + & & + & - & & & & + & & + & + & & - & - & & & + & & & \\
\hline & S6 & & - & & + & + & & & + & + & & - & & & & + & + & & & & - & + \\
\hline & S7 & & & & + & + & & & & & & - & - & - & & + & & & & & & \\
\hline & S8 & & & & - & & & & & - & & & & & & - & & & & & & \\
\hline \multirow{6}{*}{ T6 } & S3 & & & \multirow{3}{*}{-} & & & & & & & & & & - & & - & & & & & & \\
\hline & S4 & & & & & & & & & - & & & + & + & & & - & & & & & \\
\hline & S5 & & & & & - & & - & & & & & & + & & & & & & & & - \\
\hline & S6 & & & & & & & & & & - & & - & - & - & + & & & & & - & - \\
\hline & S7 & & & + & & + & & & & + & & & & - & & & & + & & & & + \\
\hline & S8 & & & & & & & & & & & & & & & - & & & & & & \\
\hline
\end{tabular}

Note 1: Item numbers correspond to Table 2.

Note 2: Plus (+) and minus (-) express a deviation more than $+/-5 \%$, and blank cells express a deviation less than $+/-5 \%$ (even).

\subsection{Distribution Patterns of Clusters in Motor} Performance

Regarding cluster analysis, the first main cluster, "physique and jumping" (PJ; Cluster A), included the following items: "Height"; "Standing broad jump"; "Weight"; "Sit-and-reach"; "Jumping back and forth";
"Side jumps"; "Mini-putt golf"; and "Vertical jump." The second-cluster, "manipulation skills" (MS; Cluster B) included: "Kick for distance"; "Rolling hoop"; "Tennis ball throw"; "Overhead throw with both hands"; and "T-ball." The third-cluster, "running and agility (RA; Cluster C) included: "25-m run"; "Stand up and dash"; "Total reaction time";"Jump over and crawl 


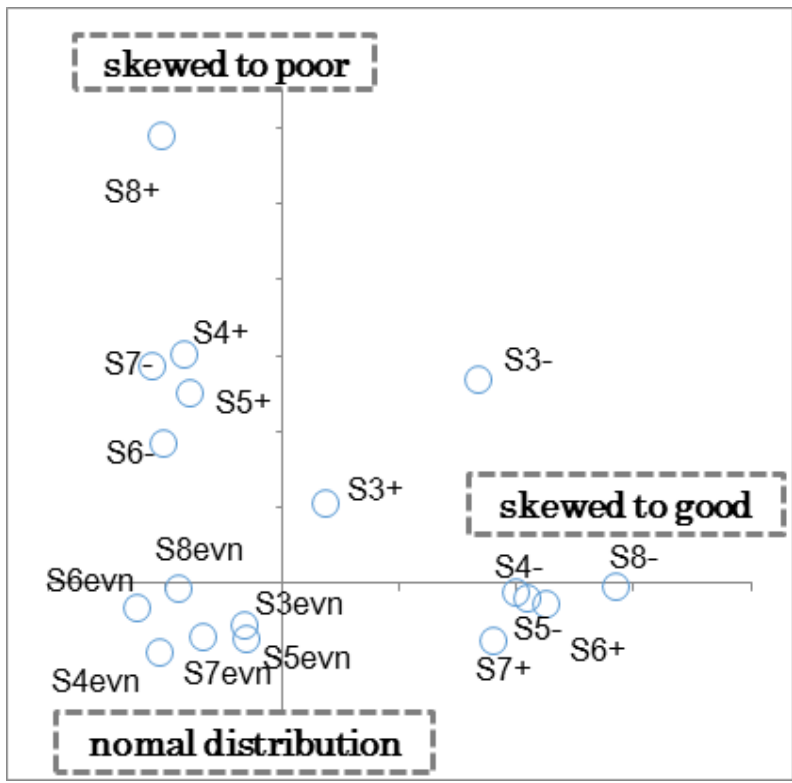

Fig. 2 Plot of category scores after rotation.

under"; "Ken-ken-pa"; "Hurdle run"; "Potato race"; and "Balance beam walk." Sub-clusters based on the three main-clusters were also investigated. PJ (Cluster A) was further divided into "height and jumping" (HJ; A1), "weight and flexibility" (WF; A2), and "jumping and control" (JC; A3). MS (Cluster B) was further divided into "kicking and rolling" (KR; B) and "throwing skills" (TS; B2). RA (Cluster C) was further divided into "running straight and agility" (RSA; C1) and "running not straight" (RNS; C2).

A scatter plot of both dimensions of CSs after rotation and mean OSs regarding PMP clusters is shown in Fig. 3. Based on the results, PJ showed a normal distribution, while MS was "skewed to poor", and RA was "skewed to good." Regarding sub-clusters, KR (B1) tended to be "skewed to poor" compared with TS (B2) in MS, and RNS (C2) tended to be "skewed to good" compared with RSA (C1) in RG.

\subsection{Gender Differences and Term Changes in the Distribution Patterns of PMP Clusters}

Gender differences and term changes in distribution patterns were examined using Hotelling's T2 test for the means of OSs in the first and second dimensions. No significant differences were found between boys and girls in $\mathrm{PJ}(\mathrm{T} 2 \mathrm{o}=5.549, \mathrm{Fo}=2.745, \mathrm{df}=[2,93]$, $P=0.069)$ or MS $(\mathrm{T} 2 \mathrm{o}=2.119, \mathrm{Fo}=1.041, \mathrm{df}=[2$, 57], $P=0.360$ ); however, a significant difference was found in RG $(\mathrm{T} 2 \mathrm{o}=8.280, \mathrm{Fo}=4.096, \mathrm{df}=[2,93], P$ $=0.020)$. Regarding gender differences in the RA sub-cluster, the generalized Mahalanobis distance of RSA (C1) was 0.593 and that of RNS (C2) was 0.311, which indicated a medium distance in both cases. Regarding seasonal changes, no significant difference was found in PJ (T2o $=4.885, \mathrm{Fo}=2.416, \mathrm{df}=[2,93]$, $P=0.095)$ or MS $(\mathrm{T} 2 \mathrm{o}=0.020, \mathrm{Fo}=0.010, \mathrm{df}=[2$, 57], $P=0.990$ ); however, a significant difference was found in RA $(\mathrm{T} 2 \mathrm{o}=8.193, \mathrm{Fo}=4.053, \mathrm{df}=[2,93], P$ $=0.021)$. The generalized Mahalanobis distances of RSA (C1) and RNS (C2) in RA were 0.476 and 0.388 , respectively, which indicated a medium distance. These results are shown in Fig. 4.

\section{Discussion}

\subsection{PMP Distribution Patterns}

Based on the clusters of the distributions for 21 PMP items based on deviations from normal, the following three features were evident: (1) PJ did not deviate from and was close to showing a normal distribution; (2) MS was "skewed to poor", with many plots "slightly poorer than the mean" and a few "slightly better than the mean"; and (3) RA was "skewed to good", with many plots "slightly better than the mean" and "slightly worse than mean". Kasuga et al. [12] examined histograms of physique and motor abilities in childhood through visual inspection and reported that the distribution of a $25-\mathrm{m}$ run skewed positively (to the right side), a standing broad jump was symmetrical, and a softball throw skewed negatively (to the left side). In Kasuga's report, "skewed to the right side" meant excellent physique and motor abilities, while "skewed to the left side" meant poor PMP. The findings in the present study support those reported by Kasuga et al. In addition, Ikeda and Aoyagi [10] studied the shape of the distributions using skewness and kurtosis statistics 


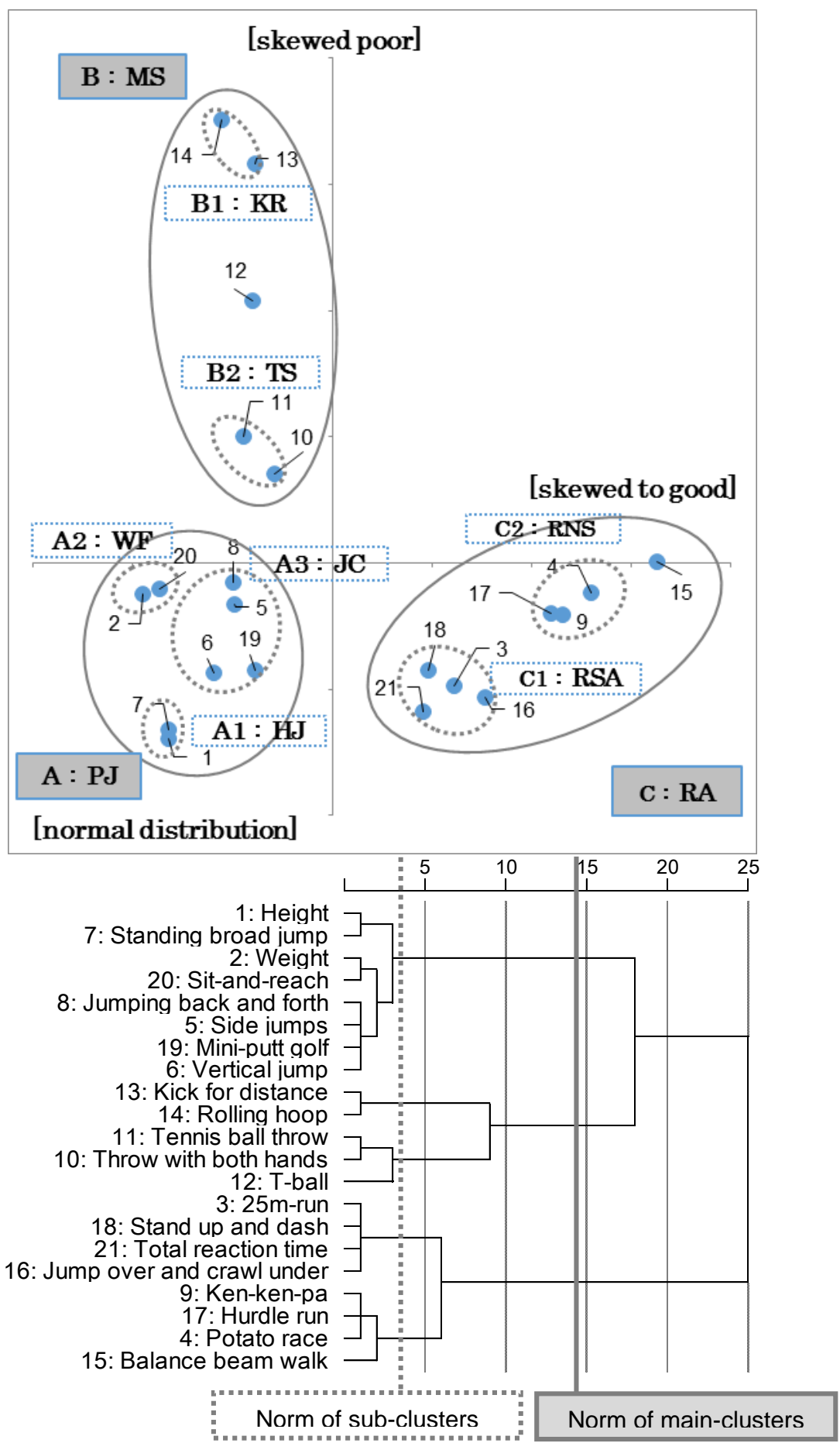

Fig. 3 Plot of clusters and test items and dendrogram.

regarding motor ability in childhood. They found that the distribution of physique and strength showed a normal pattern, that of locomotor performance with speed skewed to good, and that of manipulation/stability movements with endurance skewed to poor. The results of these previous studies investigating distributions using skewness and kurtosis were similar to those in present study. If human attributes distribute normally [5], and the skewness of distribution shows group abnormalities [6], 


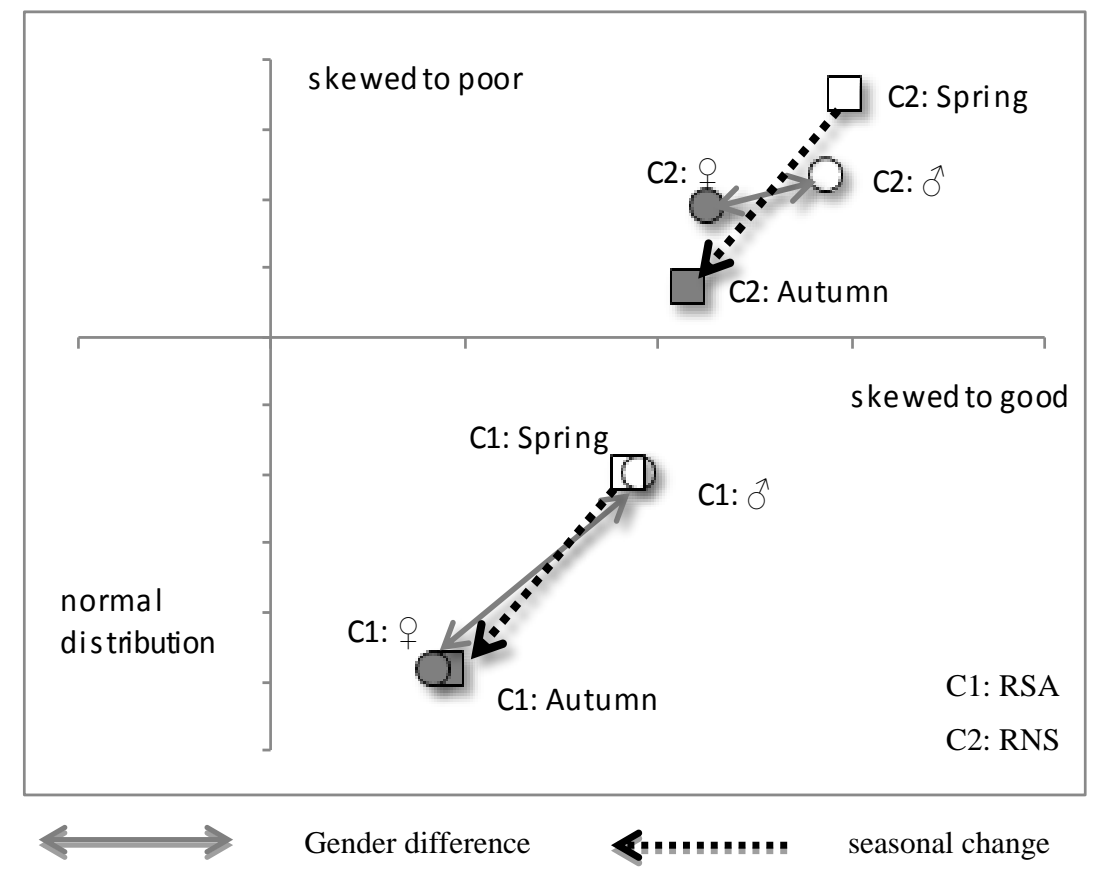

Fig. 4 Gender difference and seasonal change in sub-clusters.

these features of distributions seem to indicate that differentials in motor ability are an issue in relation to recent declines in physical fitness among children [1]. In other words, as previously mentioned, "the dropouts and elites in sport and exercise are produced by an extreme withdraw from physical activities and by excessive promotion to children in the home" $[10$, $23,24]$. Therefore, the deviation of distributions caused by RA skewed slightly to the excellent side while MS skewed to the poor side. Based on Fig. 3, more skewed deviations were found in "kicking and rolling (B1)" than in "throwing skills (B2)", and in RSA (C1) than in RNS (C2) in the motor performance clusters. This is thought to be explained by the fact that the differentials of motor ability were remarkable in complicated motor performance.

\subsection{Gender Differences in Distribution Patterns}

A gender difference was detected in RA. The gender difference in its sub-clusters, RSA (C1) and RNS (C2), was shown to be within the limit of medium range on generalized Mahalanobis distance. This result suggests that while gender differences were found in the distribution pattern of running skills, no remarkable difference was found in running course features such as straight and curved. Furthermore, as seen in Fig. 4, the distributions for boys were more skewed to the good side than that of girls in both of the running-related skills. That is, the deviations of the distributions for boys were more remarkable than those of girls, which were close to a normal distribution. In childhood, which is less marked by the effect of sexual hormones than older years [25], a substantial gender difference in motor performance is evident [10]. One of the reasons for this is thought to be at least partially associated with the physical activities that children do and the community expectations toward children during this time [26]. Based on the activities characterized by gender and adults' expectations of children, excessive activities for children causes them to become elites, but those who cannot keep up with the others become dropouts; this is considered particularly remarkable for boys. On the other hand, few extreme elites or dropouts were found among girls; however, even in childhood, remarkable differences are seen in 
the amount of physical activities, indicating that girls are affected less than boys [14, 27]. Childhood obesity due to inactivity among children is becoming increasingly worse [28]. This may be explained by the fact that RA, which represents locomotor performance with speed, is distributed poorly in obese children, and this creates a vicious circle between obesity caused by infrequent physical activity and a decline of physical activity levels, especially in girls.

\subsection{Seasonal Changes in Distribution Patterns}

Regarding changes in distribution patterns between spring and autumn, as well as gender, a half-year difference was found in RA. When inspecting the half-year differences in RA sub-clusters, RSA (C1) and RNS (C2) were within a medium range on generalized Mahalanobis distance. This can be explained by the fact that while seasonal changes can be seen in the distribution of fast-running ability, no remarkable differences can be seen in running course features such as straight or curved. Moreover, when examining half-year changes, both RA (C1) and RNS (C2) tended to skew to good side in spring more frequently than in autumn. Hanawa [13] studied seasonal factors regarding the number of steps in children and reported a higher number in June than in January. Furthermore, Sugiura et al. [14] found that children take more steps in spring and summer than in autumn and winter. The measurements in this study were conducted in May for spring and in November for autumn. In RA, representing locomotor movements with speed, poor groups, who show improvement due to increased physical activity in the summer, have close to a normal distribution in autumn. The differences among children in being highly motivated to participate in physical activities and exercise are not as evident in winter due to the decrease in physical activities; however, these differences produce dropouts in the spring. This is thought to explain the tendency to be skewed to good.
Although Hanawa [13] did not find any seasonal differences in the number of hours children spent in elementary school, Sugiura et al. [14] and Nakano et al. [27] reported that $70 \%$ and $50 \%$, respectively, of children's total daily steps were taken while at kindergarten. It is therefore possible that large differentials in children's physical activities arose at home rather than in kindergarten, where children participate in the same activities. Differences in motor ability before starting kindergarten are thought to depend on the care children receive at home [29]. These results suggest the need for increased efforts to maintain an adequate amount of physical activity in children.

\section{Conclusion}

The disparities in motor performance seen among children appear to be the result of various features, including gender and age differences and seasonal changes. For improved growth and development, increased efforts are needed to ensure that children are engaging in appropriate levels of physical activity.

\section{Acknowledgments}

This work was supported by the Japan Society for the Promotion of Science (JSPS) under Grants-in-Aid for Scientific Research (KAKENHI) Multi-year Fund (C) Number 23500677.

\section{References}

[1] Central Council for Education. 2002. "Synthetic Plan for Improvement in Children's Physical Fitness." Tokyo: Ministry of Education, Culture, Sports, Science and Technology. Accessed October 1, 2016. http://www.mext.go.jp/b_menu/shingi/chukyo/chukyo0/t oushin/021001.htm (in Japanese)

[2] Naito, H. 2006. "For Developing Physical Fitness in Children." The Monthly Journal of the Ministry of Education, Culture, Sports, Science and Technology 1569, 32-5. (in Japanese)

[3] Nishijima, T., Kokudo, S., and Ohsawa, S. 2003. "Changes over the Years in Physical and Motor Ability in Japanese Youth in 1964-97." International Journal of Sport and Health Science 1 (1): 164-70. 
[4] Nishijima, T., Nakano, T., Takahashi, S., Suzuki, K., Yamada, H., Kokudo, S., and Ohsawa, S. 2003. "Relationship between Changes over the Years in Physical Ability and Exercise and Sports Activity in Japanese Youth." International Journal of Sport and Health Science 1 (1): 110-8.

[5] Matsuura, Y. 1989. "Trends and Problems Regarding Studies on Growth and Development." Japan Journal of Human Growth and Development Research 17: 129-30. (in Japanese)

[6] Ohshima, M. 1975. "Distribution: In Medical Studies." The Japanese Journal of Behavior Metrics 3 (1): 58-64. (in Japanese)

[7] The Committee on the Establishment of Exercise Guidelines in Young Childhood. 2012. "Exercise Guidelines in Young Childhood." The Ministry of Education, Culture, Sports, Science and Technology. Accessed October $\quad 1, \quad 2016$. http://www.mext.go.jp/a_menu/sports/undousisin/131977 1.htm (in Japanese)

[8] Sugihara, T., Kondo, M., Mori, S., and Yoshida, I. 2006. "Chronological Change in Preschool Children's Motor Ability Development in Japan from the 1960s to the 2000s." International Journal of Sport and Health Science 4: 49-56.

[9] Hands, B. 2008. "Changes in Motor Skill and Fitness Measure among Children with High and Low Motor Competence: A Five-Year Longitudinal Study." Journal of Science and Medicine in Sport 11 (2): 155-62.

[10] Ikeda, T., and Aoyagi, O. 2011. "Consideration of Bipolarization of Motor Competence Based on Deviation from the Normal Distribution in Early Childhood." Japan Journal of Human Growth and Development Research 53: 23-35. (in Japanese)

[11] Ikeda, T., and Aoyagi, O. 2013. "Bipolarization of Motor Performance and Its Relationship with Gender Differences, Age, Physical Fitness, Movement Skills and Promptness of Performance in Preschool-Aged Children." Journal of the Faculty of Integrated Human Studies and Social Sciences, Fukuoka Prefectural University 22 (2): 21-34. Accessed October 1, 2016. http://www.fukuoka-pu.ac.jp/kiyou/mokuji.htm (in Japanese)

[12] Kasuga, K., Nakano, T., and Fukutomi, K. 2013. "Individual Differences in Physical Fitness and Motor Ability among Young Children: Focus on the of Distribution Change with Aging." Journal of Health, Physical Education and Recreation 63 (2): 161-73. (in Japanese)

[13] Hanawa, S. 2011. "An Examination of the Appropriate Levels of Physical Activity on the Number of Walking Steps: Relationship between Walking Steps and Both
Variable Factors (Regular Exercise, Lifestyle) and a non-Variable Factor (Seasonal Factors)." Japan Journal of Human Growth and Development Research 54: 1-10. (in Japanese)

[14] Sugiura, H., Kinoshita, H., and Fujimoto, T. 2012. "Survey of the Seasonal Step Counts of Children." The Journal of Child Health 71 (2) 242-9. Accessed October 1, 2016. http://ci.nii.ac.jp/naid/10030559398 (in Japanese)

[15] Ikeda, T., and Aoyagi, O. 2009. "Relationships between Gender Difference in Motor Performance and Age, Movement Skills and Physical Fitness among 3- to 6-Years Old Japanese Children Based on Effect Size Calculated by Meta-Analysis." School Health 5: 9-23. Accessed October $\quad 1,2016$. http://www.shobix.co.jp/sh/tempfiles/journal/2009/031.p df

[16] Matsuura, Y. 2002. "The Growth and Development by Statistics.” Fukaido-shuppan: Tokyo. (in Japanese)

[17] Gallahue, D. L., and Donnelly, F. C. 2003. Development Physical Education for all Children 4th ed. Champaign, IL: Human Kinetics.

[18] Ikeda, T., and Aoyagi, O. 2008. "Relationship between Test Characteristics and Movement Patterns, Physical Fitness, and Measurement Characteristics: Suggestions for Developing New Test Items for 2- to 6-Year-Old Children." Human Performance Measurement 5: 9-22. Accessed October $\quad 1, \quad 2016$. http://www.shobix.co.jp/hpm/tempfiles/journal/2008/07J 041.pdf

[19] Hair, J. F., Black, W. C., Babin, B. J., and Anderson, R. E. 2010. Multivariate Data Analysis: A Global Perspective. Boston, MA: Pearson Education.

[20] Cohen, J. 1992. "A Power Primer." Psychological Bulletin 112 (1): 155-9.

[21] Thomas, J. R., and French, K. E. 1985. "Gender Differences Across Age in Motor Performance: A Meta-Analysis." Psychological Bulletin 98 (2): 260-82.

[22] Thomas, J. R., and French, K. E. 1986. "The Use of Meta-Analysis in Exercise and Sport: A Tutorial." Research Quarterly for Exercise and Sport 57 (3): 196-204.

[23] Ebihara, O. 2008. "The Space Required for Physical Activity in Children." Journal of Health, Physical Education and Recreation 58 (9): 610-16. (in Japanese)

[24] Toshima, H. 2006. "Trends in Sports and Exercise Activity in Children." Journal of Health, Physical Education and Recreation 56 (5): 344-8. (in Japanese)

[25] Matsuura, Y. 1982. The Development of Physical Fitness. Asakura-shoten: Tokyo. (in Japanese)

[26] Malina, R. M., and Bouchard, C. 1991. Growth, Maturation, and Physical Activity. Champaign, IL: Human Kinetics. 

Distribution Curves for Physique and Motor Performance in Preschool-Aged Japanese Children

[27] Nakano, T., Kasuga, K., and Murase, T. 2010. "An Examination of Appropriate Physical Activity Level in Relation to Lifestyle and Motor Ability for Kindergarten Children." Japan Journal of Human Growth and Development Research 46: 49-58. (in Japanese)

[28] Hara, M. 2009. "Are Current Children's Body Undermined?" Japan Journal of Children and Growth and Development Research 7 (2): 107-12. (in Japanese)

[29] Kasuga, K. 2009. "The Longitudinal Change of Difference in Physical Fitness Levels among Young Children: Based on the Data from a Follow-up Survey Administered over a Three-year Period." Japan Journal of Human Growth and Development Research 41: 17-27. (in Japanese) 